\title{
Generalized Logistic Regression Models Using Neural Network Basis Functions Applied to the Detection of Banking Crises
}

\author{
P.A. Gutierrez ${ }^{1}$, S. Salcedo-Sanz ${ }^{2}$, M.J. Segovia-Vargas ${ }^{3}$, A. Sanchis ${ }^{4}$, \\ J.A. Portilla-Figueras ${ }^{2}$, F. Fernández-Navarro ${ }^{1}$, and C. Hervás-Martínez ${ }^{1}$ \\ 1 Universidad de Córdoba, Córdoba, Spain \\ pagutierrez@uco.es \\ 2 Universidad Complutense de Madrid, Madrid, Spain \\ sancho.salcedo@uah.es \\ 3 Universidad de Alcalá, Alcalá de Henares, Madrid, Spain \\ 4 Bank of Spain, Madrid, Spain
}

\begin{abstract}
The financial system plays a crucial role in economic development. Financial crises are recurrent phenomena in modern financial systems. The literature offers several definitions of financial instability, but it is well known that a financial crisis with a banking crisis is the most common example of financial instability. In this paper we introduce a novel model for detection and prediction of crises, based on the hybridization of a standard logistic regression with Product Unit (PU) neural networks and Radial Basis Function (RBF) networks. These hybrid approaches are described in the paper, and applied to the detection and prediction of banking crises by using a large database of countries in the period 1981 to 1999. The proposed techniques are shown to perform better than other existing statistical and artificial intelligence methods for this problem.
\end{abstract}

\section{Introduction}

The recent financial collapse has stressed the crucial role of the financial system to guarantee economic development. The financial system is the responsible for the allocation of resources over time and among different alternatives of investment, by pricing the postposition of consumption (free risk rate) and pricing the risk (risk premium). In the last twenty years at least ten countries have experienced the simultaneous onset of banking and currency crisis, with contractions in Gross Domestic Product of between 5\% and 12\% in the first year of the crisis, and negative or only slightly positive growth for several years thereafter [1]. This emphasizes the fact that preserving financial stability is one of the main goals for policy makers from the beginning of the monetary systems. It is the especial role that banks play in the financial system and their specificities as money issuers that explain why a great number of financial crises have had the banking sector as protagonist.

N. García-Pedrajas et al. (Eds.): IEA/AIE 2010, Part III, LNAI 6098, pp. $1-10,2010$.

(C) Springer-Verlag Berlin Heidelberg 2010 
Several previous works have analyzed different episodes of banking crisis occurred. Most of these works consist of case studies, many of them applying econometric analysis of different situations. For example in 2] an econometric model is used to predict bank failures using Mexican data for the period 1991-95. In a more recent work [3], the behavior of a number of macroeconomic variables in the months before and after a banking crisis is analyzed. Thus, the authors try to identify variables that act as "early warning signals" for crises. Other studies apply classical statistical techniques such as discriminant, logit or probit analysis 4 15. However, although the obtained results have been satisfactory, all these techniques present the drawback that they make some assumptions about the model or the data distribution that are not usually satisfied. So in order to avoid these disadvantages of statistical methods, it has been recently suggested in the economic field the use of Soft-computing techniques, mainly neural networks or evolutionary computation algorithms.

In recent years, neural networks have been successfully applied to bankruptcy prediction 6 67. In the majority of cases, multilayer perceptrons (MLPs) have been applied [8], due to its simple architecture yet powerful problem-solving ability. However, alternatives to MLP have arisen in the last few years, which has not been fully tested in bankruptcy. Product Unit Neural Network (PUNN) models are an alternative to MLPs and are based on multiplicative neurons instead of additive ones. They correspond to a special class of feed-forward neural network introduced by Durbin and Rumelhart 9. Networks that make use of Product Units (PUs) have the added advantage of increased information capacity 9]. Another interesting alternative to MLPs are Radial Basis Function Neural Networks (RBFNNs). RBFNNs can be considered a local approximation procedure, and the improvement in both its approximation ability as well as in the construction of its architecture has been note-worthy [10. RBFNNs have been used in the most varied domains, from function approximation to pattern classification, time series prediction, data mining, signals processing, and nonlinear system modeling and control, but again there are very few works testing this model in bankruptcy or crisis prediction.

In this paper we consider the hybridization of these novel networks (PUs and RBFs) with a standard logistic regression to improve the performance of the classifiers in the problem of bank crises prediction. Logistic Regression (LR) has become a widely used and accepted method of analysis of binary or multi-class outcome variables as it is flexible and it can predict the probability for the state of a dichotomous variable (in our case, the probability of crisis) based on the predictor variables (macroeconomic variables in this case). The hybridization of LR and PUNNs or RBFNNs is done following the model of classifier construction given in [11, where Evolutionary Algorithms (EAs) are used to obtain the best structure of the classifiers. In this paper we show that the hybrid models involving LR, PUNNs and RBFNNs outperforms several other existing classification techniques in the problem of banking crises prediction, and they are therefore a very interesting tool to take into account in this field. 
The structure of the rest of the paper is as follows: section 2 describes in detail the hybrid models (LR-PUNNs and LR-RBFNNs) proposed in this paper. Section 3 presents the experimental section of the paper, in which we test the good performance of the proposed approaches in a Financial Crisis Database, formed by a sample of data of 79 countries in the period 1981-1999. The results are also discussed in this section. Finally, the paper is closed with some remarks and conclusions in section 4.

\section{Description of the Hybrid Methodologies Proposed}

The hybrid models we analyze in this paper for the prediction of banking crises are Generalized Logistic Regression models based on the hybridization of the standard linear model and non-linear terms. These non-linear models are constructed with basis functions obtained from Evolutionary Product Unit Neural Networks (EPUNNs) and Evolutionary Radial Basis Function Neural Networks (ERBFNNs). In this section, we describe the main characteristics of the binary LR approach and the hybrid models considered. Note that a complete description of the EPUNNs and ERBFNNs is not carried out due to space reasons (the reader can check the references 111213 for a complete description of the models and the EA used to optimize these models).

\subsection{Binary Logistic Regression (LR)}

Typically, in supervised classification, a set of $n_{\mathrm{T}}$ training samples $\left(\mathbf{x}_{1}, y_{1}\right), \ldots$, $\left(\mathbf{x}_{n_{\mathrm{T}}}, y_{n_{\mathrm{T}}}\right)$ is given. The inputs $\mathbf{x}_{i}$ (i.e. the set of macroeconomic and financial variables) form a feature space $\mathbf{X}$, and the output $y_{i}$ (i.e. the bankrupt class) has a class label $c$, which belongs to a finite set $\mathbf{C}$. A classification rule is designed based on the training data, so that, given a new input $\mathbf{x}_{i}$ with the corresponding values for the macroeconomic variables, a class $c$ from $\mathbf{C}$ with the smallest probability of error is assigned to it.

In this paper the situation considered is the following: a binary outcome variable $y$ (bankrupt or non-bankrupt) is observed together with a vector $\mathbf{x}_{i}=$ $\left(1, x_{i 1}, x_{i 2}, \ldots, x_{i k}\right)$ of covariates for each of the $n_{\mathrm{T}}$ training samples (assuming that the vector of inputs includes the constant term 1 to accommodate the intercept). The two-class is coded via a $0 / 1$ response $y_{i}$, where $y_{i}=1$ for a bankrupt sample and $y_{i}=0$ for non-bankrupt samples. Let $p$ be the conditional probability associated with the first class. Logistic Regression (LR) [14] is a widely used statistical modeling technique in which the probability $p$ of the dichotomous outcome event is related to a set of explanatory variables $\mathbf{x}$ in the form:

$$
\operatorname{logit}(p)=\ln \left(\frac{p}{1-p}\right)=f_{\mathrm{LR}}(\mathbf{x}, \boldsymbol{\beta})=\boldsymbol{\beta}^{\mathrm{T}} \mathbf{x}
$$

where $\boldsymbol{\beta}=\left(\beta_{0}, \beta_{1}, \ldots, \beta_{k}\right)$ is the vector of the coefficients of the model, $\boldsymbol{\beta}^{\mathrm{T}}$ is the transpose vector and $f_{\mathrm{LR}}(\mathbf{x}, \boldsymbol{\beta})$ is the linear function of the LR model. We 
refer to $p /(1-p)$ as odds-ratio and to the expression (11) as the log-odds or logit transformation. A simple calculation in (11) shows that the probability of occurrence of an event as a function of the covariates is non-linear and is given by:

$$
p(\mathbf{x} ; \boldsymbol{\beta})=\frac{e^{\boldsymbol{\beta}^{\mathrm{T}} \mathbf{x}}}{1+e^{\boldsymbol{\beta}^{\mathrm{T}} \mathbf{x}}} .
$$

The complementary event probability can therefore be obtained as $(1-p(\mathbf{x} ; \boldsymbol{\beta}))$. Once the conditional probability function defined in (2) is known, the Bayesian (optimal) decision rule can be constructed:

$$
r(\mathbf{x})=\operatorname{sign}\left\{\ln \left(\frac{p(\mathbf{x} ; \boldsymbol{\beta})}{1-p(\mathbf{x} ; \boldsymbol{\beta})}\right)\right\} .
$$

Given the set of macroeconomic variables $\mathbf{x}$ for a specific bank, the probability $p$ that the bank belongs to the first class can be determined from (2). Similar to the maximum-likelihood classification, these class probabilities for each new bank may be outputted to produce a soft classification. The results from this paper advocate the utility of the LR as a potential approach for the soft classification similar to other recent approaches such as the MLP neural networks or the decision tree regression. A hard classification can be produced by assigning the class having a maximum probability (in our case, as a binary outcome variable is considered, we can simply check if the probability $p$ is greater or lower than the value 0.5$)$. Observe that LR not only constructs a decision rule but it also finds a function that for any input vector defines the probability $p$ that the vector $\mathbf{x}$ belongs to the first class.

Let $D=\left\{\left(\mathbf{x}_{l}, y_{l}\right) ; 1 \leq l \leq n_{\mathrm{T}}\right\}$ be the training data set. Here it is assumed that the training sample is a realization of a set of independent and identically distributed random variables. The unknown regression coefficients $\beta_{i}$, which have to be estimated from the data, are directly interpretable as log-odds ratios or, in term of $\exp \left(\beta_{i}\right)$, as odds ratios. The log-likelihood used as the error function is:

$$
l(\boldsymbol{\beta})=\sum_{l=1}^{n_{\mathrm{T}}} y_{l} \log p\left(\mathbf{x}_{l} ; \boldsymbol{\beta}\right)+\left(1-y_{l}\right) \log (1-p(\mathbf{x} ; \boldsymbol{\beta})) .
$$

The estimation of the coefficient vector $\boldsymbol{\beta}$ is usually carried out by means of an iterative procedure like the Newton-Raphson algorithm or the Iteratively Re-weighted Least Squares (IRLS) [15].

\subsection{Logistic Regression Using Initial Covariates and Product Units (LRIPU) and Radial Basis Functions (LRIRBF)}

These two hybrid methods consider evolutionary optimization to obtain a PUNN or a RBFNN structure and input-to-hidden layer weights accurate enough. When these are obtained, a multilogistic regression maximum likelihood (ML) optimization is applied over the basis functions (PUs or RBFs) of the NN selected, 
considering also the initial covariates $\mathbf{x}$ of the problem. So their generic expression is given by:

$$
f(\mathbf{x}, \boldsymbol{\theta})=\alpha_{0}+\sum_{i=1}^{k} \alpha_{i} x_{i}+\sum_{j=1}^{m} \beta_{j} B_{j}\left(\mathbf{x}, \mathbf{w}_{j}\right)
$$

where $\boldsymbol{\theta}=(\boldsymbol{\alpha}, \mathbf{W}), \boldsymbol{\alpha}=\left(\alpha_{0}, \alpha_{1}, \ldots, \alpha_{k}, \beta_{1}, \ldots, \beta_{m}\right)$ and $\mathbf{W}=\left(\mathbf{w}_{1}, \mathbf{w}_{2}, \ldots, \mathbf{w}_{m}\right)$, with $\mathbf{w}_{j}=\left(w_{j 1}, w_{j 2}, \ldots, w_{j k}\right), w_{j i} \in R$. The coefficients $\mathbf{W}$ are given by the EA, they not being adjusted by the ML method (the reader can check the references 111213 for a complete description of this EA). The ML method only optimizes the linear part of the model, i.e. the $\boldsymbol{\alpha}$ coefficients.

The ML algorithm considered is the SimpleLogistic algorithm [16, which incrementally constructs the model and applies cross-validation, resulting in an automatic covariate selection. We obtain then two different hybrid methods: SimpleLogistic Regression using Initial Covariates and PUs (LRIPU) and SimpleLogistic Regression using Initial Covariates and RBFs (LRIRBF).

\section{Computational Experiments and Results}

In this section, we describe first the Database used to evaluate the performance of our hybrid approaches. Several existing algorithms for comparison purposes are described in subsection 3.1 Finally, the main results obtained with the proposed techniques are presented in subsection 3.2 and subsection 3.3 includes some discussion about these results.

The Financial Crisis Database used in this study is formed by a sample of 79 countries in the period 1981-1999 (annual data). The binary dependent variable is:

- Systemic and non-systemic banking crises dummy: equals one during episodes identified as in [17]. They present information on 117 systemic banking crises (defined as much or all of bank capital being exhausted) that have occurred since the late 1970s in 93 countries and 51 smaller non-systemic banking crises in 45 countries during that period. The information on crises is cross-checked with that of [5] and with International Monetary Fund staff reports and financial news.

The independent variables are the following: Monetary policy strategies, Central Bank Independence, Inflation, Real Interest Rate, Net Capital Flows to GDP, Real GDP per capita in 1995 US dollars, Real GDP growth, World Real GDP growth, Domestic Credit growth, Bank Cash to total assets, Bank Foreign Liabilities to Foreign Assets and Previous crises. More information about these variables can be obtained in 18 .

\subsection{Alternative Statistical and Artificial Intelligence Methods Used for Comparison Purposes}

Different state-of-the-art statistical and artificial intelligence algorithms have been implemented for comparison purposes. Specifically, the results of the 
following algorithms have been compared with the soft-computing techniques presented in this paper:

1. The Logistic Model Tree (LMT) [16] classifier.

2. The C4.5 classification tree inducer [19].

3 . The $k$ Nearest Neighbor $(k$-NN) classifier.

4. The Support Vector Machine (SVM) classifier [15] with RBF kernels and using the Sequential Minimal Optimization (SMO) algorithm.

5. A Gaussian Radial Basis Function Network (RBFNet) [20, deriving the centers and width of hidden units using $k$-means, and combining the outputs obtained from the hidden layer using logistic regression.

6. The Multilayer Perceptron (MLP) neural network [8], trained with a simple BackPropagation (BP) algorithm.

7. The Naive Bayes standard learning algorithm (NaiveBayes) [21.

8. The Rough Set methodology for classification [22].

These algorithm have been selected for comparison since they are some of the best performing algorithms of recent literature on classification problems. Many of these approaches have also been tested before in bankruptcy detection problem. For the LRIPU and LRIRBF methods, the experimental design was conducted using a 10-fold cross-validation procedure, with 10 repetitions per each fold. For the other methods, the results have been obtained performing 10 times a 10-fold cross validation, because all are deterministic methods, i.e. they are not based in random values and return the same result for each execution. The comparison measure is the accuracy on the generalization set or Correctly Classified Rate $\left(C C R_{\mathrm{G}}\right)$.

\subsection{Results}

In this section we compare the proposed LRIPU and LRIRBF methods with the alternative artificial intelligence methods summarized above. Table 1 shows the results obtained with all these different techniques, and the result obtained by the LRIPU and LRIRBF networks. Note that the LRIPU obtains the best result in terms of $C C R_{\mathrm{G}}$ over all techniques compared. The difference in $C C R_{\mathrm{G}}$ is really significant with respect to techniques such as Naive Bayes learning, SVM/SMO, MLP, RBFNet or the $k$-NN approach. Other techniques obtain closer results in terms of $C C R_{\mathrm{G}}$, note that the Rough Set approach is the second best technique over all the compared approaches, obtaining a result slightly better than the LRIRBF, and slightly worse than the LRIPU algorithm. The LRIRBF is the third best algorithm after LRIPU and Rough Set methodology.

In order to determine if there are significant differences in the mean results obtained, an ANOVA statistical study has also been carried out. A preliminary Ftest has been applied for assessing if there are significant differences between the mean $C C R_{\mathrm{G}}$ corresponding to the distinct methodologies, obtaining a $p$-value of 0.001 lower than the critical value $\alpha=0.05$, what means that there are significant differences between the mean obtained by the methods compared. Then a multiple comparison test has been applied to rank the different methods, previously 
Table 1. Statistical results (Mean and Standard Deviation, SD) of the $C C R_{\mathrm{G}}$ of the hybrid methods proposed compared to those obtained using different statistical and artificial intelligence methods and ranking of the different methods based on the Tamhane test. The best quantitative result method is represented in bold face.

\begin{tabular}{lc}
\hline & $C C R_{\mathrm{G}}$ \\
\hline Method & Mean $\pm \mathrm{SD}$ \\
\hline LMT & $76.09 \pm 5.23$ \\
C4.5 & $76.35 \pm 5.38$ \\
$k$-NN & $74.17 \pm 5.29$ \\
SVM/SMO & $70.29 \pm 2.34$ \\
SLogistic & $76.49 \pm 4.66$ \\
RBFNet & $70.27 \pm 4.38$ \\
MLP & $74.58 \pm 5.52$ \\
MLogistic & $76.30 \pm 4.84$ \\
NaiveBayes & $69.16 \pm 2.23$ \\
Rought sets & $77.0 \pm 3.42$ \\
\hline LRIRBF & $76.88 \pm 3.03$ \\
LRIPU & $\mathbf{7 7 . 1 7} \pm 3.59$ \\
\hline Ranking & $\mu_{\text {LRIPU } *} \geq \mu_{\text {RoughSets }} \geq \mu_{\text {LRIRBF }} \geq$ \\
& $\geq \mu_{\text {SLogistic }} \geq \mu_{\mathrm{C} 4.5} \geq \mu_{\text {MLogistic }} \geq$ \\
& $\geq \mu_{\text {LMT }} \geq \mu_{\mathrm{MLP}}>\mu_{\text {SMO }} ;$ \\
& $\mu_{\text {SMO }} \geq \mu_{\text {RBFNet }} \geq \mu_{\text {NaiveBayes }} ;$ \\
& $\mu_{\text {MLogistic }}>\mu_{\text {MLP }}$ \\
\hline
\end{tabular}

evaluating whether the equality of variances can be assumed using the Levene's test. This equality can not be assumed ( $p$-value $>0.05$ for all methods), so the multiple comparison test of Tamhane is applied. The last row of Table11includes the final ranking obtained by this test, where $\mu_{\mathrm{A}} \geq \mu_{\mathrm{B}}$ is used for indicating that methodology A yields better results than methodology $\mathrm{B}$, but the differences are not significant, and $\mu_{\mathrm{A}}>\mu_{\mathrm{B}}$ is used for indicating that methodology A yields better results than methodology B with significant differences in mean. From the observation of this ranking, it can be concluded that the LRIPU* obtain higher mean accuracy but non significant differences when compared to Rough set, SLogistic, C4.5, MLogistic, LMT and MLP methods and the differences favouring LRIPU* are significant when compared to the other methods.

In general, these results show that the proposed hybrid approaches based on $\mathrm{LR}$ and $\mathrm{PU}$ or RBF networks are robust approaches to tackle the prediction of banking crises, and obtain better results than the majority of the existing alternative methods.

\subsection{Discussion}

This section include some discussion about the models obtained. The economical interpretation of the model is based on the output of the LRIPU, shown in Table 2. In this table, the best model obtained by using the LRIPU methodology 
Table 2. Probability expression of the best LRIPU model, $C C R_{\mathrm{G}}$ value and testing confusion matrix associated to this model

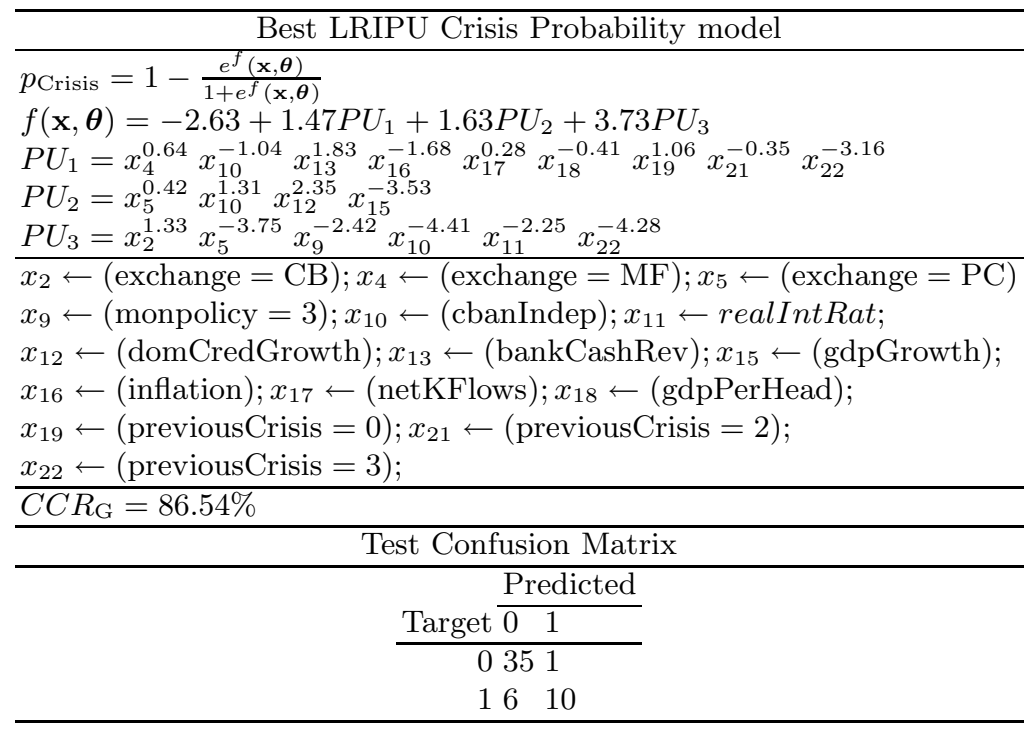

is presented. This model includes $3 \mathrm{PU}$ basis functions and 23 coefficients. At the bottom of the table, the corresponding confussion matrix for the generalization set is included, where it can be observed that only 7 banks are incorrectly classified. This is the model that shows the best performance in terms of classification. In this model is possible to see that the most important set of variables is represented by the PU3 node, followed by the other two nodes with a similar explicatory power. We focus the discussion then on the PU3 node: it shows that the combination of a currency regime close to a pegged regime instead of a currency board regime, a long lasting crisis (the country is on crisis for more than three years), a flexible monetary policy (in which objective function does not enter monetary aggregates neither inflation targets), an independent central bank, and high real interest rates positively contribute to an increment of the probability of being in crisis next year. The length of the crisis has a positive contribution in terms of the likelihood to remain in crisis, as expected. Indeed, each additional year in crisis affects more than proportionally consumer and investors confidence in absence of policies (or a positive external shock) that helps to recover it. Regarding the currency policy, the currency regime could be used to recover the confidence in the economy through stabilizing the level of prices by borrowing credibility from other countries with more stable economies. The success of this strategy will depend on the credibility in maintaining the commitments that each regime implies. The currency board regime appears as superior in terms of getting out of the crisis as a pegged regime. Both regimes reduce the level of autonomy of the national policies, but the former is more much restrictive than the later. Therefore it seems that the pegged regime is not 
a commitment strong enough to anchor agent's expectations because they still leave some room for policy maker's discretion. In addition, a flexible design of the monetary policy does not help to make this commitment credible. The design of the monetary policy needs to be perfectly aligned with the currency regime in order for the framework to be consistent. Otherwise the currency commitment would become unsustainable. This is why the independence of the central bank is not a good feature and does not appear in this node. The stabilization policy does not depend on internal monetary policy objectives but on currency policy objectives. Therefore, a dependent central bank likely does not interfere with the currency objectives that need mainly a strong political support. Finally, in this situation, the higher the interest rates, the more likely we are in crisis in the next period, because is more difficult to finance any project.

\section{Conclusions}

In this paper we have applied several hybrid algorithms mixing Logistic Regression with Product Unit neural networks (PUNNs) or Radial Basis Function networks (RBFNNs) for detecting and predicting banking crises. Both hybrid approaches consist of the evolutionary training of a PUNN or a RBFNN using an evolutionary programming approach. These hybrid models have been shown to be very strong in the problem of bank crisis prediction. In this paper we have tested these hybrid approaches in a Financial Crisis Database, formed by macroeconomic variables of 79 countries in the period 1981-1999, and the corresponding crisis/non-crisis decision variable. The results obtained have proven the good performance of the proposed approaches, that improve the results of other existing statistical and artificial intelligence techniques in the problem.

\section{Acknowledgement}

This work has been partially supported by Universidad de Alcalá and Comunidad de Madrid under grant number CCG07-UAH/AMB-3993, by the TIN 200806681-C06-03 project of the Spanish Inter-Ministerial Commission of Science and Technology (MICYT), FEDER funds and by the P08-TIC-3745 project of the "Junta de Andalucía" (Spain).

\section{References}

1. Hanson, J.: Postcrisis challenges and risks in East Asia and Latin America. In: Caprio, et al. (eds.) Financial Crisis (2006)

2. González-Hermosillo, B.: Banking sector fragility and systemic sources of fragility. International Monetary Fund, working paper 12 (1996)

3. Kaminsky, G., Reinhart, C.: The twin crises: the causes of banking and balanceof-payments problems. American Economic Review 89(3), 473-500 (1999)

4. Pasiouras, F., Tanna, S.: The prediction of bank acquisition targets with discriminant and logit analyses: Methodological issues and empirical evidence. Research in International Business and Finance 24(1), 39-61 (2009) 
5. Doma, I., Martínez-Peria, M.S.: Banking crises and exchange rate regimes: is there a link? The World Bank, working paper 2489 (2000)

6. Falavigna, G.: An analysis of the key-variables of default risk using complex systems. International Journal of Business Performance Management 10(2-3), 202-230 (2008)

7. Charalambous, C., Charitou, A., Kaourou, F.: Comparative Analysis of Artificial Neural Network Models: Application in Bankruptcy Prediction. Annals of Operations Research 99(1-4), 403-425 (2000)

8. Bishop, C.M.: Neural networks for pattern recognition. Oxford University Press, Oxford (1995)

9. Durbin, R., Rumelhart, D.: Products Units: A computationally powerful and biologically plausible extension to backpropagation networks. Neural Computation 1(1), 133-142 (1989)

10. Bishop, C.M.: Improving the generalization properties of radial basis function neural networks. Neural Computation 3(4), 579-581 (1991)

11. Hervás-Martínez, C., Martínez-Estudillo, F.J., Carbonero-Ruz, M.: Multilogistic Regression by means of Evolutionary Product-Unit Neural Networks. Neural Networks 21(7), 951-961 (2008)

12. Gutiérrez, P.A., Hervás-Martínez, C., Carbonero, M., Fernández, J.C.: Combined Projection and Kernel Basis Functions for Classification in Evolutionary Neural Networks. Neurocomputing 72(13-15), 2731-2742 (2009)

13. Martínez-Estudillo, A.C., Martínez-Estudillo, F.J., Hervás-Martínez, C., GarcíaPedrajas, N.: Evolutionary product unit based neural networks for regression. Neural Networks 19(4), 477-486 (2006)

14. Hosmer, D., Lemeshow, S.: Applied logistic regression. Wiley Interscience, Hoboken (2000)

15. Hastie, T., Tibshirani, R., Friedman, J.: The Elements of Statistical Learning. Springer, Heidelberg (2001)

16. Landwehr, N., Hall, M., Frank, E.: Logistic Model Trees. Machine Learning 59(1-2), 161-205 (2005)

17. Caprio, G., Klingebiel, D.: Episodes of systemic and borderline financial crises, dataset mimeo. The World Bank (2003)

18. Sanchis, A., Segovia-Vargas, M.J., Gil, J.A., Heras, A., Villar, J.L.: Rough Sets and the role of the monetary policy in financial stability (macroeconomic problem) and the prediction of insolvency in insurance sector (microeconomic problem). European Journal of Operational Research 181(3), 1554-1573 (2007)

19. Quinlan, J.R.: C4.5: Programs for Machine Learning. Morgan Kaufmann, San Francisco (1993)

20. Nabney, I.T.: Efficient Training of RBF Networks for Classification. International Journal of Neural Systems 14(3), 201-208 (2004)

21. Duda, R.O., Hart, P.E., Stork, D.G.: Pattern Classification, 2nd edn. Wiley Interscience, Hoboken (2000)

22. Slowinski, R., Zopounidis, C.: Application of the rough set approach to evaluatio of bankruptcy risk. International Journal of Intelligent Systems in Accounting, Finance and Management 4(1), 27-41 (1995) 